Clinical Image

\section{A rare case of abdominal pain and hematuria from retroaortic left renal vein}

\section{Pérez J* and Pozo D}

Radiology Department. Hospital Clínico Quirúrgico "Hermanos Ameijeiras", Medical Science University of Havana, Cuba

A 62-year-old female with a history of arterial hypertension, attended the emergency department due to pain in the left flank. On physical examination no showed signs of peritoneal irritation. Urinalysis was indicated, that reported microscopic hematuria and negative abdominal ultrasound. Then urotomography was performed, which confirmed the presence of retroaortic renal vein with a slight tortuous path near to renal pelvis (Figure 1).

Conservative treatment with analgesics and outpatient control by urology is treated. Four days later, the pain improved spontaneously as did hematuria. The formation of this rare variant occurs in the ventral portion of the sub supra and intercardinal anastomosis or in its dorsal part or the presence of a persistent cardinal vein. This vascular variant can generate compression between the aorta and the anterior aspect of the vertebral body and can cause abdominal pain in the left flank with or without hematuria. The CT contributes to its diagnosis.

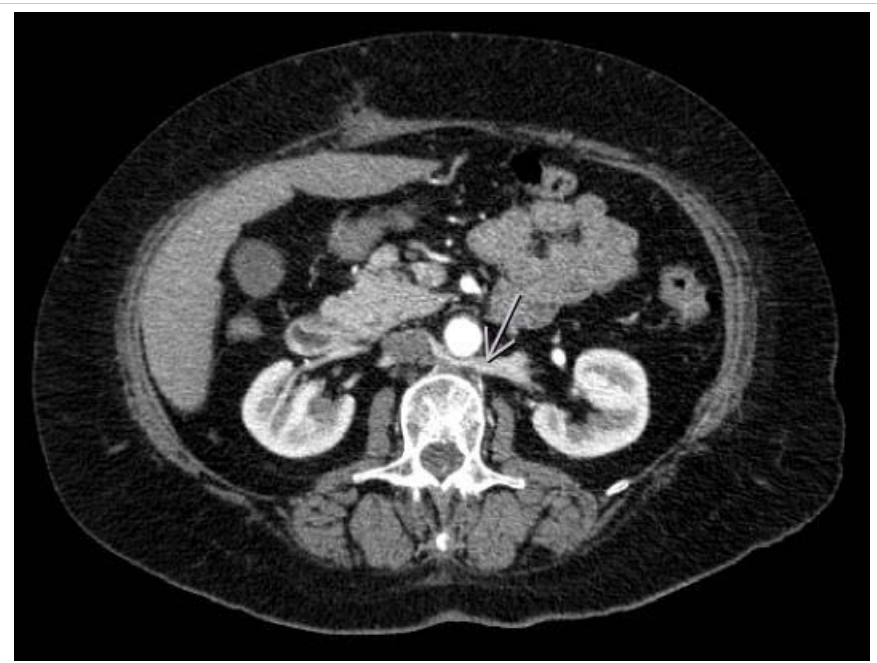

Figure 1: Urotomography in venous phase- Retroaortic renal vein with a slight tortuous path near to renal pelvis (arrow).

\section{More Information}

*Address for Correspondence: Pérez J, Radiology Department, Hospital Clínico Quirúrgico

"Hermanos Ameijeiras" Calle San Lázaro \#701 esq. a Belascoaín, Piso 5, Centro Habana, Havana, Cuba, Email: jperezrmd@gmail.com

Submitted: 13 December 2019

Approved: 16 December 2019

Published: 17 December 2019

How to cite this article: Pérez J, Pozo D. A rare case of abdominal pain and hematuria from retroaortic left renal vein. J Clin Med Exp Images. 2019; 3: 012-012.

DOI: dx.doi.org/10.29328/journal.jcmei.1001013

Copyright: @ 2019 Pérez J, et al. This is an open access article distributed under the Creative Commons Attribution License, which permits unrestricted use, distribution, and reproduction in any medium, provided the original work is properly cited.

(D) Check for updates

OPEN ACCESS 